\title{
Two Approaches to the Dataset Interlinking Recommendation Problem
}

\author{
Giseli Rabello Lopes ${ }^{1}$, Luiz André P. Paes Leme ${ }^{2}$, \\ $\underline{\text { Bernardo Pereira Nunes }}^{1}$, Marco Antonio Casanova ${ }^{1}$, and $\underline{\text { Stefan Dietze }}^{3}$ \\ 1 Department of Informatics, Pontifical Catholic University of Rio de Janeiro, \\ Rio de Janeiro, RJ - Brazil, CEP 22451-900 \\ \{grlopes, bnunes, casanova\}@inf .puc-rio.br \\ 2 Computer Science Institute, Fluminense Federal University, \\ Niterói, RJ - Brazil, CEP 24210-240 \\ lapaesleme@ic.uff.br \\ 3 L3S Research Center, Leibniz University Hannover, Appelstr. 9a, \\ 30167 Hannover, Germany \\ \{dietze\}@13s.de
}

\begin{abstract}
Whenever a dataset $t$ is published on the Web of Data, an exploratory search over existing datasets must be performed to identify those datasets that are potential candidates to be interlinked with $t$. This paper introduces and compares two approaches to address the dataset interlinking recommendation problem, respectively based on Bayesian classifiers and on Social Network Analysis techniques. Both approaches define rank score functions that explore the vocabularies, classes and properties that the datasets use, in addition to the known dataset links. After extensive experiments using real-world datasets, the results show that the rank score functions achieve a mean average precision of around $60 \%$. Intuitively, this means that the exploratory search for datasets to be interlinked with $t$ might be limited to just the top-ranked datasets, reducing the cost of the dataset interlinking process.
\end{abstract}

Keywords: Linked Data, data interlinking, recommender systems, Bayesian classifier, social networks.

\section{Introduction}

Over the past years there has been a considerable movement towards publishing data on the Web following the Linked Data principles [1. According to those principles, to be considered 5-star, a dataset must comply with the following requirements: (i) be available on the Web; (ii) be available as machine-readable structured data; (iii) be in a non-proprietary format; (iv) use open standards from W3C (i.e. RDF and SPARQL) to identify resources on the Web; and (v) be linked to other people's data to provide additional data. This paper addresses the last requirement.

Briefly, in the context of Linked Data, a dataset is a set of RDF triples. A resource identified by an RDF URI reference $s$ is defined in a dataset $t$ iff $s$ occurs as the subject of a triple in $t$. 
A feature of a dataset is a vocabulary URI, a class URI or a property URI used in triples of the dataset. One may then represent the dataset by one or more of its features.

Let $t$ and $u$ be two datasets. A link from $t$ to $u$ is a triple of the form $(s, p, o)$ such that $s$ is defined in $t$ and $o$ is defined in $u$. We say that $t$ is linked to $u$, or that $u$ is linked from $t$, iff there is at least one link from $t$ to $u$. We also say that $u$ is relevant for $t$ iff there is at least one resource defined in $u$ that can be linked from a resource defined in $t$.

The dataset interlinking recommendation problem can then be posed as follows:

Given a finite set of datasets $\boldsymbol{D}$ and a dataset t, compute a rank score for each dataset $u \in \boldsymbol{D}$ such that the rank score of $u$ increases with the chances of $u$ being relevant for $t$.

To address the dataset interlinking recommendation problem, this paper proposes and compares two approaches respectively based on Bayesian classifiers and on Social Network link prediction measures. Both approaches define rank score functions that explore the dataset features and the known links between the datasets. The experiments used real-world datasets and the results show that the rank score functions achieve a mean average precision of around $60 \%$. Intuitively, this means that a dataset interlinking tool might limit the search for links from a dataset $t$ to just the top ranked datasets with respect to $t$ and yet find most of the links from $t$.

The rest of the paper is organized as follows. Section 2 discusses related work. Section 3 introduces our proposed approaches based on Bayesian classifiers and on Social Network Analysis techniques. Section 4 presents the experiments conducted to test and compare the approaches. Finally, Section 5 contains the conclusions and directions for future work.

\section{Related Work}

In this paper, we extend previous work 23 that introduced preliminary versions of the rank score functions respectively based on the Bayesian and the Social Network approaches. This paper contains significantly new results over our previous work in so far as it (i) explores different sets of features to compute rank score functions; (ii) uses modified rank score functions to interlink new datasets without known links; and (iii) provides a comprehensive comparison of the approaches using different feature sets.

In more detail, the paper improves previous results as follows. As for the Bayesian ranking definition, the paper formally defines how to manage the lack of observations of co-occurrences between features and links. Without this new definition, null probabilities could lead the score function to a discontinuity region $(\log (0))$. In the SN-based ranking definition, we propose a new score function (not defined in $[3]$ ) which combines preferential attachment and resource allocation measures. The definition of the similarity set of the target dataset is also novel. 
Furthermore, we do not assume that one knows the existing links of the dataset to which one wants to generate recommendations for. This assumption is realistic for new datasets and tackles one of the core challenges of the Linked Data principles. Indeed, for new datasets, the approach proposed in 3 will not work and that presented in 2 will generate recommendations based only on the popularity of the datasets (i.e., the recommendations will be the same for all datasets).

We explored different sets of features - properties, classes and vocabularies - to compute the rank score functions. Moreover, we thoroughly compared the performance of the improved approaches using different feature sets.

Nikolov et al. 415 propose an approach to identify relevant datasets for interlinking, with two main steps: (i) searching for potentially relevant entities in other datasets using as keywords a subset of labels in the new published dataset; and (ii) filtering out irrelevant datasets by measuring concept similarities obtained by applying ontology matching techniques.

Kuznetsov 6 describes a linking system which is responsible for discovering relevant datasets for a given dataset and for creating instance level linkage. Relevant datasets are discovered by using the referer attribute available in the HTTP message header, as described in 17, and ontology matching techniques are used to reduce the number of pairwise comparisons for instance matching. However, this work does not present any practical experiments to test the techniques.

When compared with these approaches, the rank score functions proposed in this paper use only metadata and are, therefore, much simpler to compute and yet achieve a good performance.

The next set of papers aim at recommending datasets with respect to user queries, which is a problem close, but not identical to the problem discussed in this paper. Lóscio et al. 8 address the recommendation of datasets that contribute to answering queries posed to an application. Their recommendation function estimates a degree of relevance of a given dataset based on an information quality criteria of correctness, schema completeness and data completeness. Wagner et al. 9 also propose a technique to find relevant datasets for user queries. The technique is based on a contextualization score between datasets, which is in turn based on the overlapping of sets of instances of datasets. It uses just the relationships between entities and disregards the schemas of the datasets. Oliveira et al. 10 use application queries and user feedback to discover relevant datasets. Application queries help filter datasets that are potentially strong candidates to be relevant and user feedback helps analyze the relevance of such candidates.

Toupikov et al. 11 adapt the original PageRank algorithm to rank existing datasets with respect to a given dataset. The technique uses the Linksets descriptions available in VoID files as the representation of relationships between datasets and the number of triples in each Linkset as the weight of the relationships. Results show that the proposed technique performs better than traditional ranking algorithms, such as PageRank, HITS and DRank. As the rank score functions defined in this paper, the version of the PageRank algorithm the authors propose depends on harvesting VoID files. 


\section{Ranking Techniques}

Sections 3.1 and 3.2 introduce two approaches to compute rank score functions, leaving a concrete example to Section 3.3 .

\subsection{Bayesian Ranking}

This section defines a rank score function inspired on conditional probabilities. However, we note that the rank score is not a probability function. We proceed in a stepwise fashion until reaching the final definition of the rank score function, in Equation 9.

Let $\boldsymbol{D}$ be a finite set of datasets, $d_{i}$ be a dataset in $\boldsymbol{D}$ and $t$ be a dataset one wishes to link to datasets in $\boldsymbol{D}$. Let $T$ denote the event of selecting the dataset $t, D_{i}$ denote the event of selecting a dataset in $\boldsymbol{D}$ that has a link to $d_{i}$, and $F_{j}$ denote the event of selecting a dataset that has feature $f_{j}$ (recall that a feature of a dataset is a vocabulary URI, a class URI or a property URI used in triples of the dataset).

We tentatively define the rank score function as a conditional probability:

$$
\text { score }_{0}\left(d_{i}, t\right)=P\left(D_{i} \mid T\right)
$$

that is, score $_{0}\left(d_{i}, t\right)$ is the conditional probability that $D_{i}$ occurs, given that $T$ occurred. As required, this score function intrinsically favors those datasets with the highest chance of defining links from $t$.

We then rewrite score $_{0}$, using Bayes's rule, as follows:

$$
\text { score }_{1}\left(d_{i}, t\right)=\frac{P\left(T \mid D_{i}\right)}{P(T)} P\left(D_{i}\right)
$$

As in Bayesian classifiers 1213 , by representing $t$ as a bag of features $\boldsymbol{F}=$ $\left\{f_{1}, \ldots, f_{n}\right\}$, one may rewrite score $_{1}$ as:

$$
\text { score }_{2}\left(d_{i}, t\right)=\frac{P\left(\left\{f_{1}, \ldots, f_{n}\right\} \mid D_{i}\right)}{P\left(\left\{f_{1}, \ldots, f_{n}\right\}\right)} P\left(D_{i}\right)
$$

By the naive Bayes assumption $1213, P\left(\left\{f_{1}, f_{2}, \ldots, f_{n}\right\} \mid D_{i}\right)$ can be computed by multiplying conditional probabilities for each independent event $F_{j}$ (the event of selecting datasets with just the feature $\left.f_{j}\right)$. Moreover, $P\left(\left\{f_{1}, \ldots, f_{n}\right\}\right)$ does not change the rank order because it is the same for all $d_{i}$. Hence, we remove this term. The new score function becomes:

$$
\text { score }_{3}\left(d_{i}, t\right)=\left(\prod_{j=1 . . n} P\left(F_{j} \mid D_{i}\right)\right) P\left(D_{i}\right)
$$

The final score function is obtained from score $_{3}$ by replacing the product of the probabilities by a summation of logarithms, with the help of auxiliary functions $p$ and $q$ that avoid computing $\log (0)$. 
Intuitively, the definitions of functions $p$ and $q$ penalize a dataset $d_{i}$ when no dataset with feature $f_{j}$ is linked to $d_{i}$ or when no dataset is linked to $d_{i}$. The definitions depend on choosing a constant $C$ that satisfies the following restriction (where $m$ is the number of datasets in $\boldsymbol{D}$ and $n$ is the number of features considered):

$$
\begin{aligned}
C & <\min \left(C^{\prime}, C^{\prime \prime}\right) \\
C^{\prime} & =\min \left\{P\left(F_{j} \mid D_{i}\right) \in[0,1] / P\left(F_{j} \mid D_{i}\right) \neq 0 \wedge j \in[1, n] \wedge i \in[1, m]\right\} \\
C^{\prime \prime} & =\min \left\{P\left(D_{i}\right) \in[0,1] / P\left(D_{i}\right) \neq 0 \wedge i \in[1, m]\right\}
\end{aligned}
$$

Then, $p$ is defined as follows:

$$
p\left(F_{j}, D_{i}\right)= \begin{cases}C, & \text { if } P\left(F_{j} \mid D_{i}\right)=0 \\ P\left(F_{j} \mid D_{i}\right), & \text { otherwise }\end{cases}
$$

Intuitively, $p$ avoids computing $\log \left(P\left(F_{j} \mid D_{i}\right)\right)$ when $P\left(F_{j} \mid D_{i}\right)=0$, that is, when no dataset with feature $f_{j}$ is linked to $d_{i}$. In this case, $d_{i}$ is penalized and $p\left(F_{j}, D_{i}\right)$ is set to $C$.

Likewise, $q$ is defined as follows:

$$
q\left(D_{i}\right)= \begin{cases}C, & \text { if } P\left(D_{i}\right)=0 \\ P\left(D_{i}\right), & \text { otherwise }\end{cases}
$$

Intuitively, $q$ avoids computing $\log \left(P\left(D_{i}\right)\right)$ when $P\left(D_{i}\right)=0$, that is, when no dataset is linked to $d_{i}$. In this case, $d_{i}$ is also penalized and $q\left(D_{i}\right)$ is set to $C$.

We define the final rank score function in two steps. We first define:

$$
\operatorname{score}\left(d_{i}, t\right)=\left(\sum_{j=1 . . n} \log \left(p\left(F_{j}, D_{i}\right)\right)\right)+\log \left(q\left(D_{i}\right)\right)
$$

and then eliminate $p\left(F_{j}, D_{i}\right)$ from Equation 8 :

$$
\operatorname{score}\left(d_{i}, t\right)=c\left|N_{i}\right|+\left(\sum_{f_{j} \in P_{i}} \log \left(P\left(F_{j} \mid D_{i}\right)\right)\right)+\log \left(q\left(D_{i}\right)\right)
$$

where

$$
\begin{aligned}
& -c=\log (C) \\
& -N_{i}=\left\{f_{j} \in \boldsymbol{F} / P\left(F_{j} \mid D_{i}\right)=0\right\} \\
& -P_{i}=\boldsymbol{F}-N_{i}
\end{aligned}
$$

In particular, we note that, when $t$ does not have any feature (i.e., when $n=0)$, the score function takes into account only the unconditional probability $P\left(D_{i}\right)$. In this case, the most popular datasets, such as DBpedial 1 and Geonames, will be favored by the score function at the expenses of perhaps more

\footnotetext{
1 http://dbpedia.org/

2 http://www.geonames.org/
} 
appropriate datasets. The ranking may not be accurate in such borderline cases, but a popularity-based ranking is preferable to no ranking at all, when nothing is known about $t$.

Equation 9, therefore, defines the final score function that induces the ranking of the datasets in $\boldsymbol{D}$ (from the largest to the smallest score). Section 3.3 illustrates how the score is computed.

Based on the maximum likelihood estimate of the probabilities $\underline{13}$ in a training set of datasets, the above probabilities can be estimated as follows:

$$
\begin{aligned}
P\left(F_{j} \mid D_{i}\right) & =\frac{\operatorname{count}\left(f_{j}, d_{i}\right)}{\sum_{j=1}^{n} \operatorname{count}\left(f_{j}, d_{i}\right)} \\
P\left(D_{i}\right) & =\frac{\operatorname{count}\left(d_{i}\right)}{\sum_{i=1}^{m} \operatorname{count}\left(d_{i}\right)}
\end{aligned}
$$

where $\operatorname{count}\left(f_{j}, d_{i}\right)$ is the number of datasets in the training set that have feature $f_{j}$ and that are linked to $d_{i}, \operatorname{count}\left(d_{i}\right)$ is the number of datasets in the training set that are linked to $d_{i}$, disregarding the feature set. Thus, for any dataset $t$ represented by a set of features, the rank position of each of the datasets in $\boldsymbol{D}$ can be computed using Equations 7, 9, 10 and 11.

Note that Equation [10 depends on the correlation between $f_{j}$ and $d_{i}$ in the training set. This means that the higher the number of datasets correlating feature $f_{j}$ with links to $d_{i}$, the higher the probability in Equation 10, Moreover, as Equation 4 depends on the joint probability of the features $f_{j}$ of $t$, the higher the number of features shared by $t$ and the datasets linked to $d_{i}$ with high probability, the higher score $\left(d_{i}, t\right)$ will be. That is, if the set of features of $t$ is very often correlated with datasets that are linked to $d_{i}$ and $t$ is not already linked to $d_{i}$, then it is recommended to try to link $t$ to $d_{i}$.

Finally, we stress that, if a dataset $t$ exhibits a set of features $\boldsymbol{F}$, one can choose any subset of $\boldsymbol{F}$ as the representation of $t$. Thus, each possible representation may generate different rankings with different performances and one cannot predict in advance which representation will generate the best ranking. Section 4 then compares the results obtained for several different feature sets.

\subsection{Social Network-Based Ranking}

In Social Networks Analysis (SNA), the network is typically represented as a graph, where the nodes are the entities (e.g., users, companies) and the edges are the relationships between them (e.g., follows, shares, befriends, co-authorships). In SNA, the link prediction problem refers to the problem of estimating the likelihood of the existence of an edge between two nodes, based on the already existing edges and on the attributes of the nodes 14 . We propose to analyze the dataset interlinking recommendation problem in much the same way as the link prediction problem.

As in Section 3.1, let $\boldsymbol{D}$ be a finite set of datasets, $d_{i}$ be a dataset in $\boldsymbol{D}$ and $t$ be a dataset one wishes to link to datasets in $\boldsymbol{D}$. Recall again that a feature of 
a dataset is a vocabulary URI, a class URI or a property URI used in triples of the dataset.

The Linked Data network for $\boldsymbol{D}$ is a directed graph such that the nodes are the datasets in $\boldsymbol{D}$ and there is an edge between datasets $u$ and $v$ in $\boldsymbol{D}$ iff there is a link from $u$ to $v$.

The similarity set of a dataset $t$, denoted $S_{t}$, is the set of all datasets in $\boldsymbol{D}$ that have features in common with $t$. The popularity set of a dataset $d_{i} \in \boldsymbol{D}$, denoted $P_{d_{i}}$, is the set of all datasets in $\boldsymbol{D}$ that have links to $d_{i}$.

Among the traditional measures adopted for link prediction 15|14, we will use Preferential Attachment and Resource Allocation. Indeed, the results reported in 16, which analyzed the dataset interlinking recommendation problem using just the existing links, indicate that these two measures achieved the best performance.

Preferential Attachment. The Preferential Attachment score estimates the possibility of defining a link from $t$ to $d_{i}$ as the product of the cardinality of the similarity set of $t$, denoted $\left|S_{t}\right|$, and the cardinality of the popularity set of $d_{i}$, denoted $\left|P_{d_{i}}\right|$, and is defined as follows:

$$
p a_{0}\left(t, d_{i}\right)=\left|S_{t}\right| \times\left|P_{d_{i}}\right|
$$

However, since $\left|S_{t}\right|$ is independent of $d_{i}$, this term does not influence the rank score of the datasets. Thus, we may ignore it and define $p a$ as follows:

$$
p a\left(t, d_{i}\right)=\left|P_{d_{i}}\right|
$$

Resource Allocation. Let $d_{j}$ be a dataset in $\boldsymbol{D}$, distinct from $d_{i}$. Intuitively, if there are links from $t$ to $d_{j}$ and from $d_{j}$ to $d_{i}$ and there are many other datasets that have links to $d_{j}$, then $d_{j}$ must be a generic dataset (eg. DBpedia, Geonames, etc.). Therefore, $d_{j}$ does not necessarily suggest any possible link from $t$ to $d_{i}$. On the other hand, if there are not many datasets that have links to $d_{j}$, then this might be a strong indication that $d_{j}$ is a very particular dataset for both $t$ and $d_{i}$ and, therefore, a link from $t$ to $d_{i}$ might as well be defined. Thus, the strength of the belief in the existence of a link from $t$ to $d_{i}$ increases inversely proportional to the number of datasets which have links to $d_{j}$, i.e., depends on the cardinality of the popularity set of $d_{j}$, again denoted $\left|P_{d_{j}}\right|$.

The Resource Allocation score estimates the possibility of defining a link from $t$ to $d_{i}$ as a summation of the inverse of the cardinality of the popularity set of the datasets in the intersection of the datasets linked from $t$, which is the similarity set $S_{t}$ of $t$, and the datasets linked to $d_{i}$, which is the popularity set $P_{d_{i}}$ of $d_{i}$. It is defined as follows:

$$
r a\left(t, d_{i}\right)=\sum_{d_{j} \in S_{t} \cap P_{d_{i}}} \frac{1}{\left|P_{d_{j}}\right|}
$$


Combined Score. To obtain more accurate results, we combine the two previous scores into a new score, defined as follows:

$$
\operatorname{score}\left(t, d_{i}\right)=r a\left(t, d_{i}\right)+\frac{p a\left(t, d_{i}\right)}{|\boldsymbol{D}|}
$$

This final score gives priority to the ra score; the $p a$ score, normalized by the total number of datasets to be ranked $(|\boldsymbol{D}|)$, will play a role when there is a tie or when the $r a$ value is zero. Section 4.3 comments on the adequacy of defining a combined score function.

\subsection{Example of Rank Score Computations}

We illustrate how to compute rank score functions, using both approaches, with the help of a schematic example. We selected a subset of the datasets indexed by the DataHub3, using the Learning Analytics and Knowledg 4 dataset [17, referred to as lak in what follows, as the target of the recommendation.

As features of $l a k$, we used three classes, swc:ConferenceEvent, swrc:Proceedings and swrc:InProceedings, obtained from the LinkedUp project Web site 5 .

As the candidates to be ranked, we selected the datasets webscience, webconf, wordnet, dblp and courseware. They were chosen because we considered all datasets that share at least one feature with lak (webscience and webconf) and all datasets linked from them ( wordnet and $d b l p$ ). In addition, to better illustrate the computation of the rank scores, we also considered courseware, one of the datasets linked to wordnet.

The similarity set of lak consists of the datasets webscience and webconf, since they share at least one feature with lak. The datasets webscience and webconf shares respectively the swc:ConferenceEvent class and the swc:ConferenceEvent, swrc:Proceedings and swrc:InProceedings classes with lak.

Table 1 and Table 2 respectively list the URIs of all such datasets and classes. Figure 1 depicts these objects, where the directed thin arrows represent the existing links among the datasets, the thick arrows denote links from lak to datasets in its similarity set (used only by Social Network-based approach) and the dashed lines indicate which datasets have what features. The dashed cylinders refer to groups of datasets (the number of datasets grouped is indicated inside the cylinder).

The rank score functions have to rank the datasets webscience, webconf, wordnet, $d b l p$ and courseware according to the chances of defining links from resources in lak to resources in each of these datasets. The datasets in the similarity set of lak (webscience and webconf) are included in the list of candidates to be ranked because they are not yet linked from lak.

\footnotetext{
3 http://datahub.io/

4 http://lak.linkededucation.org

5 http://linkedup-project.eu/
} 
Table 1. The dataset acronym and the corresponding URI

\begin{tabular}{|l|l|}
\hline Dataset & URI \\
\hline lak & http://lak.linkededucation.org \\
webscience & http://webscience.rkbexplorer.com \\
webconf & http://webconf.rkbexplorer.com \\
dblp & http://knoesis.wright.edu/library/ontologies/swetodblp/ \\
wordnet & http://www.w3.org/TR/wordnet-rdf \\
courseware & http://courseware.rkbexplorer.com \\
\hline
\end{tabular}

Table 2. The class feature acronym and the corresponding URI

\begin{tabular}{|l|l|}
\hline Class & URI \\
\hline swc:ConferenceEvent & http://data.semanticweb.org/ns/swc/ontology\#ConferenceEvent \\
swrc:Proceedings & http://swrc.ontoware.org/ontology\#Proceedings \\
swrc:InProceedings & http://swrc.ontoware.org/ontology\#InProceedings \\
\hline
\end{tabular}

The Social Network-based rank score function (shown in Equation 15) ranks wordnet in the first position (the largest score value), $d b l p$ in the second position, courseware in the third position and webscience and webconf (with tied scores) in the last two positions. Recall that the Social Network-based score function is the sum of two terms, $r a$ and $p a$. The first two best ranked datasets have scores determined by ra greater than zero because they are linked from webconf, which is in the similarity set of lak. The remaining datasets are ranked only by the $p a$ term, including webconf and webscience, because they are in the similarity set of $l a k$.

Using the Bayesian approach, the rank score function ranks $d b l p$ in the first position, wordnet in the second position, courseware in the third position and webscience and webconf (with tied scores) in the last two positions. It is not possible to adequately estimate probability values for webscience and webconf because they are both not linked from any other dataset. Thus, in this example, their score values will be the minimum, determined in this case by $c * 4=-60$ (omitted from the table in Figure 1 for convenience). Intuitively, the top ranking positions assigned to wordnet and $d b l p$ are justified because both datasets are linked from datasets that share some feature with lak and the popularity of both can be estimated.

A manual inspection performed in the two best ranked datasets by both approaches indicated that the recommendation of $d b l p$ is justified because the DBLP digital library 6 indexes the papers published in the LAK and EDM conferences, as does the lak dataset. Then, resources of $l a k$ can be linked to resources in $d b l p$ (e.g., using owl:sameAs property). The recommendation of wordnet is also justified because resources of $l a k$ can be linked to the corresponding concepts defined in wordnet.

Both approaches presented in the paper (SN-based and Bayesian) are related to the correlation between features and links. Therefore, our approaches could

\footnotetext{
6 http://www.informatik.uni-trier.de/ ley/db/
} 


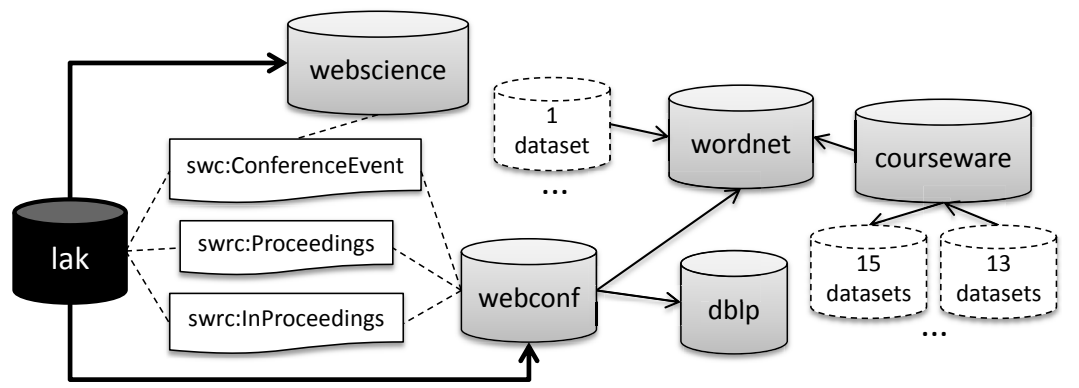

Bayesian Scores (using c=-15)

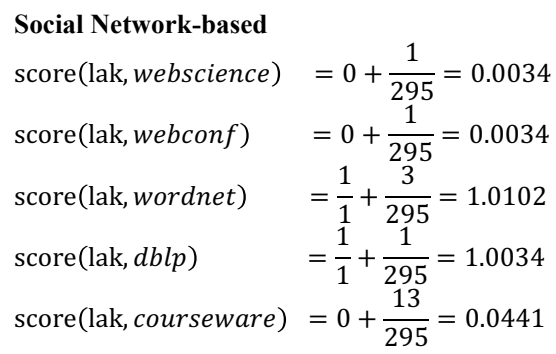

\begin{tabular}{|c|c|c|c|}
\hline Partial Result & $\mathbf{d}_{\mathbf{1}}$ & $\mathbf{d}_{\mathbf{2}}$ & $\mathbf{d}_{\mathbf{3}}$ \\
\hline count $\left(\mathrm{f}_{1}, \mathrm{~d}_{\mathrm{i}}\right)$ & 1 & 1 & 0 \\
\hline $\operatorname{count}\left(\mathrm{f}_{2}, \mathrm{~d}_{\mathrm{i}}\right)$ & 1 & 1 & 0 \\
\hline $\operatorname{count}\left(\mathrm{f}_{3}, \mathrm{~d}_{\mathrm{i}}\right)$ & 1 & 1 & 0 \\
\hline$\sum_{\mathrm{i}=1, \ldots, ., \mathrm{n}} \operatorname{count}\left(\mathrm{f}_{\mathrm{i}}, \mathrm{d}_{\mathrm{i}}\right)$ & 3 & 3 & 0 \\
\hline $\operatorname{count}\left(\mathrm{d}_{\mathrm{i}}\right)$ & 3 & 1 & 13 \\
\hline $\mathrm{P}\left(\mathrm{F}_{2} \mid \mathrm{D}_{\mathrm{i}}\right)$ & 0.33 & 0.33 & - \\
\hline $\mathrm{P}\left(\mathrm{F}_{2} \mid \mathrm{D}_{\mathrm{i}}\right)$ & 0.33 & 0.33 & - \\
\hline $\mathrm{P}\left(\mathrm{F}_{3} \mid \mathrm{D}_{\mathrm{i}}\right)$ & 0.33 & 0.33 & - \\
\hline $\mathrm{P}\left(\mathrm{D}_{\mathrm{i}}\right)$ & 0.004 & 0.001 & 0.019 \\
\hline$\left|\mathrm{N}_{\mathrm{i}}\right|$ & 0 & 0 & 3 \\
\hline $\log _{2}\left(\mathrm{P}\left(\mathrm{F}_{1} \mid \mathrm{D}_{\mathrm{i}}\right)\right)$ & -2 & -2 & - \\
\hline $\log _{2}\left(\mathrm{P}\left(\mathrm{F}_{2} \mid \mathrm{D}_{\mathrm{i}}\right)\right)$ & -2 & -2 & - \\
\hline $\log _{2}\left(\mathrm{P}\left(\mathrm{F}_{3} \mid \mathrm{D}_{\mathrm{i}}\right)\right)$ & -2 & -2 & - \\
\hline $\log _{2}\left(\mathrm{P}\left(\mathrm{D}_{\mathrm{i}}\right)\right)$ & -7.86 & -9.45 & -5.74 \\
\hline score $\left(\mathrm{d}_{\mathrm{i}}, \mathrm{t}\right)$ & $-\mathbf{1 2 . 6 1}$ & $-\mathbf{1 4 . 2 0}$ & $\mathbf{- 5 0 . 7 4}$ \\
\hline
\end{tabular}

Fig. 1. Example including the datasets links, associated features and the score computation

recommend two datasets that do not share any feature (vocabulary, class and property) as candidates to be interlinked. Considering the example, lak and $d b l p$ have completely different feature sets and yet could be interlinked. As there is webconf (that has common features with lak) linked to $d b l p$, then our approaches can recommend to try to interlink lak to $d b l p$.

\section{Experiments}

\subsection{Notation and Performance Measures}

To motivate how we define the performance measure, recall that the goal of the rank score functions is to reduce the effort required to discover new links 
from a dataset $t$. With the appropriate ranking, datasets more likely to contain links from $t$ will be better positioned in the ranking so that the search may be concentrated on the datasets at the top of the ranking. Thus, in the experiments, we evaluated the rank score functions using the Mean Average Precision, which is a traditional Information Retrieval measure 1819. Furthermore, we remark that, since the rank score functions induce a ranking of all datasets, the recall is always $100 \%$ and is, therefore, not used as a performance measure.

To define the Mean Average Precision (MAP), we adopt the following notation (recall that a dataset $u$ is relevant for a dataset $t$ iff there is at least one resource defined in $u$ that can be linked from a resource defined in $t$ ):

$-D$ is a set of datasets

- $\boldsymbol{T}$ is a set of datasets, disjoint from $\boldsymbol{D}$, one wishes to link to datasets in $\boldsymbol{D}$

$-t \in \boldsymbol{T}$

- $G_{t}$ is the set of datasets in $\boldsymbol{D}$ with known links from $t$ (the gold standard for t)

- Prec@ $k_{t}$ is the number of relevant datasets obtained until position $k$ in a ranking for $t$, divided by $k$ (the precision at position $k$ of a ranking for $t$ )

- Ave $P_{t}=\left(\sum_{k}\right.$ Prec@ $\left.k_{t}\right) /\left|G_{t}\right|$, for each position $k$ in a ranking for $t$ in which a relevant dataset occurs (the average precision at position $k$ of a ranking for $t$ )

The Mean Average Precision (MAP) of a rank score function over the datasets in $\boldsymbol{T}$ is then defined as follows:

$$
M A P=\text { average }\left\{\text { Ave } P_{t_{j}} / t_{j} \in \boldsymbol{T} \wedge\left|G_{t_{j}}\right|>0\right\}
$$

Moreover, in order to evaluate whether the improvements are statistically significant, a paired statistical Student's T-test 1819 was performed. According to Hull 20], the T-test performs well even for distributions which are not perfectly normal. We adopted the usual threshold of $\alpha=0.05$ for statistical significance. When a paired T-test obtained a $p$-value (probability of no significant difference between the compared approaches) less than $\alpha$, there is a significant difference between the compared approaches.

\subsection{Dataset}

We tested the rank score functions with metadata extracted from the DataHub catalog, a repository of metadata about datasets, in the style of Wikipedia. DataHub is openly editable and can be accessed through an API provided by the data cataloguing software CKAN7. The set of data used in our experiments is available at http://www.inf.puc-rio.br/ casanova/ Publications/Papers/2014-Papers/interlinking-test-data.zip and was extracted in April 2013.

\footnotetext{
7 http://ckan.org
} 
We adopted as features the properties, classes and vocabularies used in the datasets, in different combinations. From the DataHub catalog, we managed to obtain 295 datasets with at least one feature and 697 links between these datasets. The number of distinct features was 12,102 , where 10,303 were references to properties, 6,447 references to classes and 645 references to vocabularies; the number of relations between datasets and features was 17,395 .

We conclude with brief comments on how we extracted metadata from the DataHub catalog.

Let $t$ be a dataset and $V$ be a set of VoID descriptions 21 for $t$, available through the catalog. We extracted classes and properties used in $t$ from dataset partitions defined in $V$, using the void:class and the void:property properties. We obtained vocabularies used in $t$ from the void:vocabulary property. We uncovered links of $t$ from Linkset descriptions associated with $t$ that occur in $V$. A void:Linkset describes a set of triples $(s, p, o)$ that link resources from two datasets through a property $p$. The void:subjects Target property designates the dataset of the subject $s$ and the void:objects Target property indicates the dataset of the object $o$.

We also extracted links via the catalog API, which exposes a multivalued property, relationships, whose domain and range is the complete set of catalogued datasets. In this case, assertions of the form " $[$ relationships $]=\_$node" and "_node $[$ object $]=u$ " indicate that $t$ is linked to a dataset $u$.

\subsection{Testing Strategy}

To evaluate the performance of the rank score functions, we adopted the traditional 10-fold cross validation approach, where a testing set is randomly partitioned into 10 equally-sized subsets and the testing process is repeated ten times, each time using a different subset as a testing partition and the rest of the objects in the testing set as a training partition.

In our experiments, the 295 datasets obtained from the DataHub catalog played the role of the testing set. The 10-fold cross validation then generated 10 different pairs $\left(\boldsymbol{T}_{i}, \boldsymbol{D}_{i}\right)$, for $i=1, \ldots, 10$, of testing and training partitions. The known links between datasets in $\boldsymbol{D}_{i}$ were preserved, those between datasets in $\boldsymbol{T}_{i}$ were ignored, and those from datasets in $\boldsymbol{T}_{i}$ to $\boldsymbol{D}_{i}$ were used as the gold standard for the datasets in $\boldsymbol{T}_{i}$. Each test consisted of computing the MAP for the pair $\left(\boldsymbol{T}_{i}, \boldsymbol{D}_{i}\right)$. Then, we computed the overall average of the MAPs for the 10 tests, referred to as the overall MAP in Section 4.4 .

We used the training partition to estimate probabilities, using Equations 10 and 11, when testing the Bayesian approach, and to construct the Linked Data network, when testing the Social Network-based approach.

\subsection{Results}

This section describes the experiments we conducted to evaluate the rank score functions generated by the two approaches presented in Section 3, referred to as the Bayesian approach and the Social Network-based (SN-based) approach 
Table 3. Overall Mean Average Precision

\begin{tabular}{|c|c|c|c|c|}
\hline \multirow{2}{*}{ Approach } & \multicolumn{4}{|c|}{ Feature set } \\
\cline { 2 - 5 } & properties & classes & vocabularies & all \\
\hline SN-based & $48.46 \%$ & $\mathbf{5 7 . 1 8 \%}$ & $48.27 \%$ & $51.57 \%$ \\
Bayesian & $59.18 \%$ & $55.31 \%$ & $51.20 \%$ & $\mathbf{6 0 . 2 9 \%}$ \\
\hline
\end{tabular}

(using the rank score function defined in Equation 15). We combined each of the approaches with the following feature sets: (i) only properties; (ii) only classes; (iii) only vocabularies; and (iv) all these three features.

Table 3 depicts the overall MAP results obtained by each combination of approach and feature set. The Bayesian approach using all three features achieved the best performance; the Bayesian approach using properties obtained the second best result; and the SN-based approach using classes was the third best result. In fact, the Bayesian approach obtained better results than the SN-based approach using properties or vocabularies as single features. The worst results obtained by both approaches used vocabularies as a single feature. This probably happened because, in our experiments, we have a restrict number of references to vocabularies in the datasets.

We also calculated the overall MAP of the rank score functions based only on preferential attachment $(\mathrm{pa})$ and resource allocation $(\mathrm{ra})$, using classes as single features. We respectively obtained $43.64 \%$ and $44.75 \%$, which are lower than the overall MAP for the rank score function defined in Equation 15.

Finally, we applied a paired T-test to investigate whether there are statistically significant differences between the overall MAP results of the different approaches and selected feature sets. Table 4 shows the $p$-values obtained by all T-tests performed, where the results is boldface represent differences which are not statistically significant.

The T-test of the SN-based approaches indicate that the SN-based approach using the rank score function defined in Equation [15] and using classes as features outperforms the SN-based approaches using preferential attachment $(p a)$ or resource allocation $(\mathrm{ra})$ and classes as features.

A T-test was also performed for overall MAP results of the SN-based approaches using classes and using the other feature selections. The T-tests indicate that the SN-based approach using the rank score function defined in Equation 15 and classes achieved a statistically significant improvement when compared to all others (using properties, vocabularies and all features). Thus, there are evidences that classes are the best feature selection to be used with the SN-based approach.

For the Bayesian approach, we compared the results obtained by using all features (the configuration with the best overall MAP) with the results obtained using all other feature selections. The T-tests indicate that the overall MAP results of the Bayesian approach using all features and using only properties do not present a statistically significant difference. This suggests that using only properties is an adequate strategy to be adopted with the Bayesian approach. 
Table 4. The $p$ values applying T-test

\begin{tabular}{|l|c|c|c|c|c|}
\hline \multirow{3}{*}{ SN-based with classes } & \multicolumn{3}{|c|}{ SN-based with } & \multirow{2}{*}{ pa } & \multirow{2}{*}{ ra } \\
\cline { 2 - 4 } & properties & vocabularies & all features & & \\
\cline { 2 - 4 } & $5.26 \mathrm{E}-05$ & 0.00195 & 0.03683 & $5.46 \mathrm{E}-08$ & $1.35 \mathrm{E}-05$ \\
\hline \multirow{3}{*}{ Bayesian with all features } & \multicolumn{3}{|c|}{ Bayesian with } & \multirow{2}{*}{ SN-based with classes } \\
\cline { 2 - 4 } & properties & classes & vocabularies & \\
\cline { 2 - 4 } & $\mathbf{0 . 1 0 6 4 1}$ & 0.00408 & 0.00022 & $\mathbf{0 . 0 7 2 7 5}$ \\
\hline
\end{tabular}

We also used a paired T-test to investigate whether there is a statistically significant difference between the overall MAP values obtained by the best configuration for the SN-based approach (using classes) and the best configuration for the Bayesian approach (using all features). The T-tests indicate that there is no statistical difference between the overall MAP results of both approaches.

In conclusion, these observations indicate that the SN-based approach using classes or the Bayesian approach using properties induce the best rank score functions, since they achieve the best results and are simple to compute. This is the main result of the paper.

\section{Conclusions}

This paper compared two approaches respectively based on Bayesian classifiers and on Social Network Analysis techniques to address the dataset interlinking recommendation problem. Both approaches define rank score functions that explore only metadata features - vocabularies, classes and properties - and the known dataset links. The results show that the rank score functions achieve a mean average precision of around $60 \%$. This means that a dataset interlinking tool might use the rank score functions to limit the search for links from a dataset $t$ to just the top ranked datasets with respect to $t$ and yet find most of the links from $t$. Thus, the rank score functions are potentially useful to reduce the cost of dataset interlinking.

The computation of the rank score functions depends on harvesting metadata from Linked Data catalogs and from the datasets themselves, a problem shared by other Linked Data techniques, but they are not restricted using only VoID descriptions. This limitation in fact calls attention to the importance of harvesting metadata, that can be carried out in different ways, including the inspection of the datasets by crawlers, a problem we address elsewhere [22, to fulfill the Linked Data promises.

Finally, we plan to further improve the definition of the rank score functions. One generic strategy is to improve the network analysis-based score by considering the frequency of the schema elements. Often two datasets share similar classes and properties, but they strongly differ on the number of instances. Another aspect to explore would be feature similarity (e.g., string similarity between two features), rather than just considering the intersection of the feature sets. 
Acknowledgments. This work was partly funded by the LinkedUp project (GA No:317620), under the FP7 programme of the European Commission, by CNPq, under grants $160326 / 2012-5,303332 / 2013-1$ and $557128 / 2009$ 9, by FAPERJ, under grants E-26/170028/2008, E-26/103.070/2011 and E26/101.382/2014, and by CAPES, under grant 1410827.

\section{References}

1. Berners-Lee, T.: Linked Data. In: Design Issues. W3C (July 2006)

2. Leme, L.A.P.P., Lopes, G.R., Nunes, B.P., Casanova, M.A., Dietze, S.: Identifying candidate datasets for data interlinking. In: Daniel, F., Dolog, P., Li, Q. (eds.) ICWE 2013. LNCS, vol. 7977, pp. 354-366. Springer, Heidelberg (2013)

3. Lopes, G.R., Leme, L.A.P.P., Nunes, B.P., Casanova, M.A., Dietze, S.: Recommending tripleset interlinking through a social network approach. In: Lin, X., Manolopoulos, Y., Srivastava, D., Huang, G. (eds.) WISE 2013, Part I. LNCS, vol. 8180, pp. 149-161. Springer, Heidelberg (2013)

4. Nikolov, A., d'Aquin, M.: Identifying Relevant Sources for Data Linking using a Semantic Web Index. In: WWW2011 Workshop on Linked Data on the Web, Hyderabad, India. CEUR Workshop Proceedings, vol. 813. CEUR-WS.org (March 29, 2011)

5. Nikolov, A., d'Aquin, M., Motta, E.: What Should I Link to? Identifying Relevant Sources and Classes for Data Linking. In: Pan, J.Z., Chen, H., Kim, H.-G., Li, J., Wu, Z., Horrocks, I., Mizoguchi, R., Wu, Z. (eds.) JIST 2011. LNCS, vol. 7185, pp. 284-299. Springer, Heidelberg (2012)

6. Kuznetsov, K.A.: Scientific data integration system in the linked open data space. Programming and Computer Software 39(1), 43-48 (2013)

7. Mühleisen, H., Jentzsch, A.: Augmenting the Web of Data using Referers. In: WWW2011 Workshop on Linked Data on the Web, Hyderabad, India. CEUR Workshop Proceedings, vol. 813. CEUR-WS.org (March 29, 2011)

8. Lóscio, B.F., Batista, M., Souza, D.: Using information quality for the identification of relevant web data sources. In: The 14th International Conference on Information Integration and Web-Based Applications \& Services, IIWAS 2012, Bali, Indonesia, December 3-5, pp. 36-44. ACM, New York (2012)

9. Wagner, A., Haase, P., Rettinger, A., Lamm, H.: Discovering related data sources in data-portals. In: Proceedings of the First International Workshop on Semantic Statistics, Co-located with the the International Semantic Web Conference (2013)

10. de Oliveira, H.R., Tavares, A.T., Lóscio, B.F.: Feedback-based data set recommendation for building linked data applications. In: I-SEMANTICS 2012 - 8th International Conference on Semantic Systems, I-SEMANTICS 2012, Graz, Austria, September 5-7, pp. 49-55. ACM (2012)

11. Toupikov, N., Umbrich, J., Delbru, R., Hausenblas, M., Tummarello, G.: Ding! dataset ranking using formal descriptions. In: Proceedings of the WWW2009 Workshop on Linked Data on the Web, LDOW 2009, Madrid, Spain. CEUR Workshop Proceedings, vol. 538. CEUR-WS.org (April 20, 2009)

12. Witten, I.H., Frank, E., Hall, M.A.: Data Mining: Practical Machine Learning Tools and Techniques. Morgan Kaufmann (January 2011)

13. Manning, C.D., Schütze, H.: Foundations of Statistical Natural Language Processing. MIT Press (2002) 
14. Lü, L., Jin, C.H., Zhou, T.: Similarity index based on local paths for link prediction of complex networks. Physical Review E 80(4), 046122 (2009)

15. Liben-Nowell, D., Kleinberg, J.: The link-prediction problem for social networks. J. Am. Soc. Inf. Sci. Technol. 58(7), 1019-1031 (2007)

16. Caraballo, A.A.M., Nunes, B.P., Lopes, G.R., Leme, L.A.P.P., Casanova, M.A., Dietze, S.: Trt - a tripleset recommendation tool. In: Proceedings of the ISWC 2013 Posters \& Demonstrations Track, Sydney, Australia. CEUR Workshop Proceedings, vol. 1035, pp. 105-108. CEUR-WS.org (October 23, 2013)

17. Taibi, D., Dietze, S.: Proceedings of the LAK Data Challenge, Leuven, Belgium, April 9. CEUR Workshop Proceedings, vol. 974. CEUR-WS.org (2013)

18. Baeza-Yates, R.A., Ribeiro-Neto, B.A.: Modern Information Retrieval - the concepts and technology behind search, 2nd edn. Pearson Education Ltd., Harlow (2011)

19. Manning, C.D., Raghavan, P., Schütze, H.: Introduction to Information Retrieval. Cambridge University Press (July 2008)

20. Hull, D.: Using statistical testing in the evaluation of retrieval experiments. In: Proceedings of the 16th Annual International ACM SIGIR Conference on Research and Development in Information Retrieval, SIGIR 1993, pp. 329-338. ACM, New York (1993)

21. Alexander, K., Cyganiak, R., Hausenblas, M., Zhao, J.: Describing Linked Datasets with the VoID Vocabulary. W3C (March 2011)

22. do Vale Gomes, R., Casanova, M.A., Lopes, G.R., Leme, L.A.P.P.: A metadata focused crawler for linked data. In: Proceedings of the 16th International Conference on Enterprise Information Systems, ICEIS 2014, Lisbon, Portugal, April 27-30, vol. 2, pp. 489-500. SciTePress (2014) 